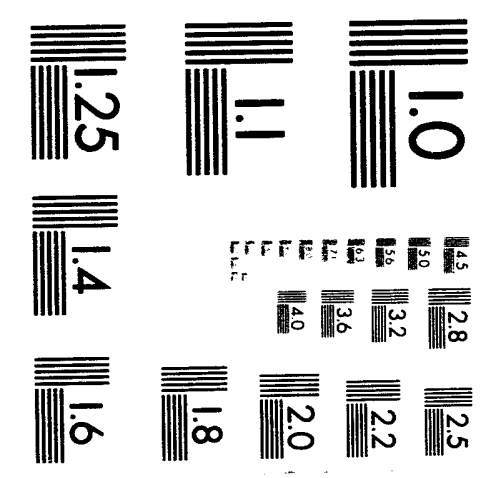



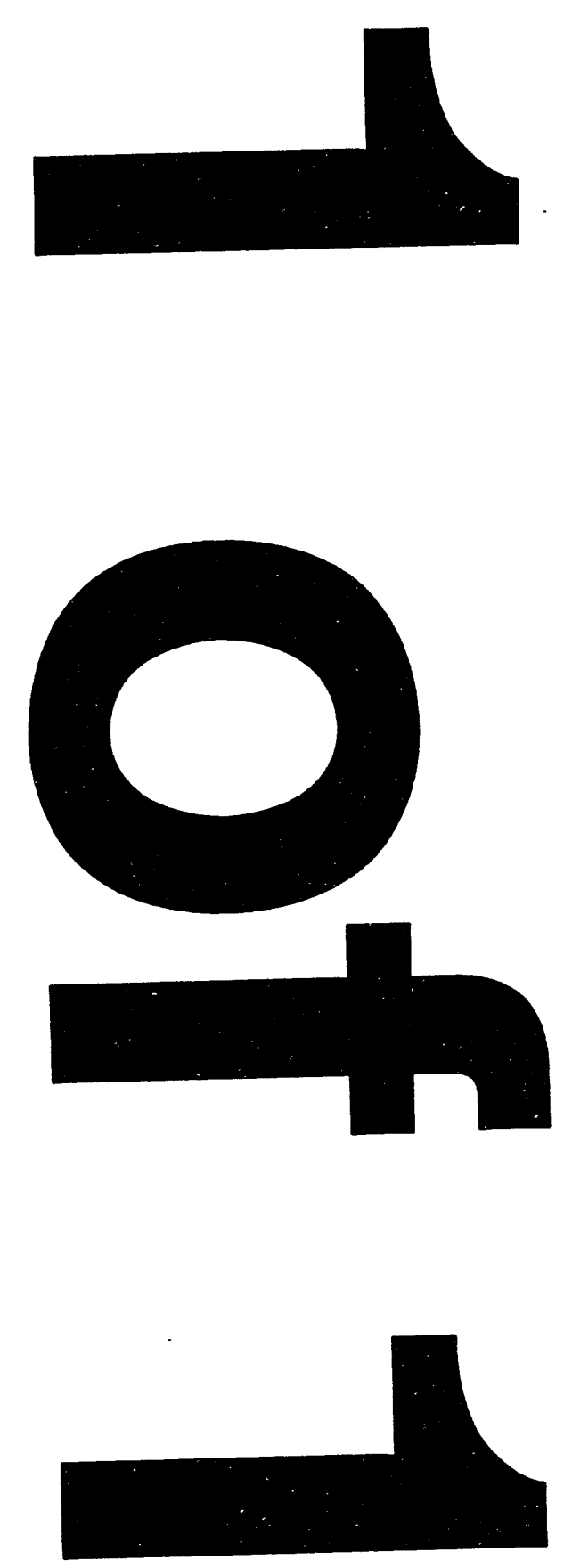
TECHNICAL PROGRESS REPORT

\title{
SUPERSONIC BARE METAL CLUSTER BEAMS
}

For Period Feb. 1, 1993 - March 31, 1994

Principal Investigator: R. E. Smalley

\section{DISCLAIMER}

\begin{abstract}
This report was prepared as an account of work sponsored by an agency of the United States Government. Neither the United States Government nor any agency thereof, nor any of their employees, makes any warranty, express or implied, or assumes any legal liability or responsibility for the accuracy, completeness, or usefulness of any information, apparatus, product, or process disclosed, or represents that its use would not infringe privately owned rights. Reference herein to any specific commercial product, process, or service by trade name, trademark, manufacturer, or otherwise does not necessarily constitute or imply its endorsement, recommendation, or favoring by the United States Government or any agency thereof. The views and opinions of authors expressed herein do not necessarily state or reflect those of the United States Government or any agency thereof.
\end{abstract}


The following is a list of papers acknowledging support from this grant, which have either appear in print or have been submitted since last year's report. Included also in this list are two U.S. Patents which have issued this past year. These two involve new methods of producing fullerenes and metallo-fullerenes that were developed with partial support of the U.S. Department of Energy through this grant.

176. "From Dopyballs to Nanowires", R. E. Smalley, Materials Science and Engineering B., 19/1-2, 1-7 (1993).

178. "The Third Form of Carbon", L.P.F. Chibante, R. E. Smalley, On Clusters and Clustering. From Atoms to Fractals. P. J. Reynolds, editor (North Holland, Amsterdam, 1993) pp. 99-115.

180. "Electronic Structure of the Hollow-cage $M_{8} X_{12}$ Clusters", L. Lou, T. Guo and R. E. Smalley, and Peter Nordlander, Journal of Chemical Physics, 99 (7), 5301 5305 (1993).

183. "Solar Generation of the Fullerenes" F.L.P. Chibante, Andreas Thess, J.M. Alford, M.A. Diener, R. E. Smalley, J. Phys. Chem., 97, 8696-8700 (1993)

185. "Electric Arc Process for Making Fullerenes" R. E. Smalley, and Robert E. Haufler, United States Patent \# 5,227,038, (1993)

187. "Photoelectron Spectroscopy of Transition Metal Clusters: Correlation of Valence Electronic Structure to Reactivity", R. T. Laaksonen, J. Conceicao, L. S. Wang, T. Guo, P. Nordlander, and R. E. Smalley, Physical Review B, Submitted ...... $(8 / 20 / 93)$

191. "Process for Making Fullerenes with Metal Inside", R. E. Smalley, US Patent No. 5,300,203. Issued April 5, 1994.

Publication \#183 listed above describes a new method for the production of fullerenes using direct solar vaporization of a carbon feedstock. This development has been the subject of a new U.S. Patent application which is currently pending. A copy of this paper is appended to this Technical Report as Appendix I. The genesis of this work was our efforts over the past year to produce fullerene-encapsulated metal atoms and metal clusters in sufficient yields, and with sufficient cleanliness to permit their isolation, manipulation, and detailed study. As is described in the paper, this work started out with experiments using carbon arcs and plasmas in a quartz tube furnace under completely air and moisture-free conditi., s. In the process we found that one of the most important processes limiting fullerene yield was photochemical activation of fullerene reactions by the ultraviolet light from the arc or plasma torch.

In thinking about how one might in principle eliminated this problem, it occured to us that the best answer would be to simply overwhelm the process, and use light itself to perform the vaporization, avoiding the production of a UV emitting plasma entirely. It further occurred to us that one could also use the intense light flux to bleach away all carbon clusters until the carbon vapor had migrated sufficiently far from the vaporization 
region, that it was safely away from the blackbody glow of this region and was sufficiently dillute and well-mixed with the desired dopant metals that fullerene production was optimized, and the product could be safely collected. The paper reproduced in Appendix I describes our initial success at a proof of principal experiment.

Unknown to us at the time, similar work was being tried by the DOE-supported laboratory, NREL, in Golden Colorado. We have since discussed the possibility of scaling this sort of experiment up to more significant sizes with the group at NREL and with Roland Winston at $U$. of Chicago. As yet no further work has been done along these lines, simply because we have been so heavily involved in our other work with metallofullerene and nanotube production and study. However, we continue to believe (for the reasons discussed in the paper) that this solar production method may very well evolve to be the only way of producing fullerenes, and particularly metallofullerenes, in truely bulk (ton) quantities. The uranium-fullerenes such as $U @ \mathrm{C}_{28}, \mathrm{Ux} @ \mathrm{C}_{60}$ and larger uranium carbide nanoparticles encased in fullerene shells may well end up being highly useful in nuclear fuel rod production and processing. Accordingly, one of the students working on this project (Michael Diener) has taken a position with Nicholas Coppa in the actinide group at Los Alamos to look into this sort of issue in a very serious way. Mr. Diener will complete his PhD research at LANL, but will remain a member of the Smalley group, and will receive his degree from Rice.

Elsewhere on the metal clusters in fullerenes front, we have gone to laser vaporization production of the doped fullerenes with completely air- and moisture-free work up of the products. Although this method will never be large scale enough to be of commercial importance, it does produce doped fullerenes in sufficient amount for most characterizations, and at the moment challenge of how one purifies and manipulates these new materials is far more pressing that the question of how one would go about producing them by the kilogram.

One of the most intriguing possibilities for future heterogeneous catalysts is to use completely new strategies for dispersing, supporting, and protecting the catalytic transition metal particles. Although it is little appreciated, the continuous graphene sheet is actually one of the most inert two-dimensional chemical surfaces known. One of the principal results of the discovery of the fullerenes is that we now realize that through the incorporation of pentagons and heptagons it is possible to wrap this sheet around any shape object one can conceive of in three dimensions. Imagine, for example, a new catalyst which consists of transition metal clusters (iron, or cobalt for example) physisorbed into dimples of controlled, nanometer diameter and depth in a graphene sheet produced not be making holes in the sheet, but rather by the encorporation of heptagons to join the flat hexagonal surface to a tubular hole, which in turn either smoothly connects (via more heptagons) to a second graphene sheet on the under side, or terminates in a hemifullerene with 6 pentagons. Alternatively, suppose the catalyst support consists of two concentric fullerene cylinders, connected to each other with out edges though the use of 6 - and 7-membered rings. Here there is the possibility that the extreme chemical inertness of the graphene lattice can provide the necessary survivability of the catalyst under real operating conditions, while the diameter and length of the buckytube pore or dimple closely controls to tm clusters size to the most optimum value, and keeps these tm clusters from sintering together.

Here, at least conceptually, there appears to be a way out of the central difficulty tm cluster research has had in having a real impact on the practical problems of catalysis. The problem has really never been that the tm clusters themselves are catalytically inactive. They are, if anything, too active. Instead the problem has been one of support, and size control. The extreme chemical inertness of continuous 
graphene sheets, coupled with what we now realize is the complete freedom one may have to conform this sheet to any desired topology on a nanometer scale may be the ultimate answer to this problem. For these reasons we have come to believe in this group that the study of various means of producing and manipulating carbon nanostructures may be critical to the future of heterogeneous catalysis.

Over half of the effort of the group has concentrated on the production of carbon nanotubes, and continuous nanofibers. For most of the year, our research in this area has been interesting, but proceeded quite slowly due to our inability to image directly the nanotubes and fibers we were producing. Last fall, however, we were able to convince Rice University to make a major commitment to nanotechnology in general. As part of this commitment Rice has purchased a new Field Emission SEM and a $200 \mathrm{kV}$ TEM, and these instruments have now been installed (as of 2 weeks ago). The students in the group have now finished their initial training on these instruments, and we are now SEEING every day, highly detailed images of nanotubes, fibers, fullerene onions, and carbon-encapsulated metal clusters. This will be critical in the next, most crucial phase of our continuous carbon fiber production research.

Up to now we have been able to demostrate that it is possible to grow continuous carbon fibers by directing a focused ion laser beam at the tip of the growing fiber while biasing it a several hundred volts in a vacuum and exposing the hot tip to hydrocarbons at low pressures. The high electric field at the tip of the fiber keeps the graphene sheet edges exposed, and these highly reactive bare edges then readily add new carbon atoms from the pyrolyzed hydrocarbon radical fragments. Our problem, however, has been that the carbon fibers thus produced are too imperfect. No matter what we have tried, we have not been able to anneal them into perfect fullerene fibers where the graphene sheets are continuous around the fiber diameter. Instead these fibers have many seams between the graphene sheets, and these seams are the site of new chemisorption that produces a thickening and branching of the already too-imperfect fiber. Our conclusion is that there is no way of solving this problem without going back to the original structure that seeded the fiber growth in the first place. We have been using commercial 8 micron diameter fibers sharpened by laser vaporization in a high electric field in the vacuum of the apparatus. Unfortunately, this always appears to seed imperfect fiber growth. Instead, now we are actively mounting individual carbon nanotubes produced in carbon arcs on the end of hypodermic needles. These perfect fullerene tubes ought to provide a perfect "seed crystal" for continuous fiber growth. Here the recently availability of FEGSEM and TEM will be critical in monitoring our progress.

Success with this project of continuous (and perfect) fullerene fiber growth will be a major watershed for future research in the production of a wide range of carbon nanostructures, including among many others, transition metal catalyst supports.

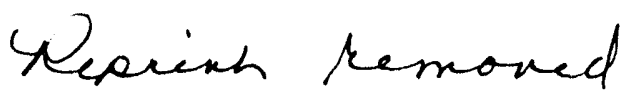



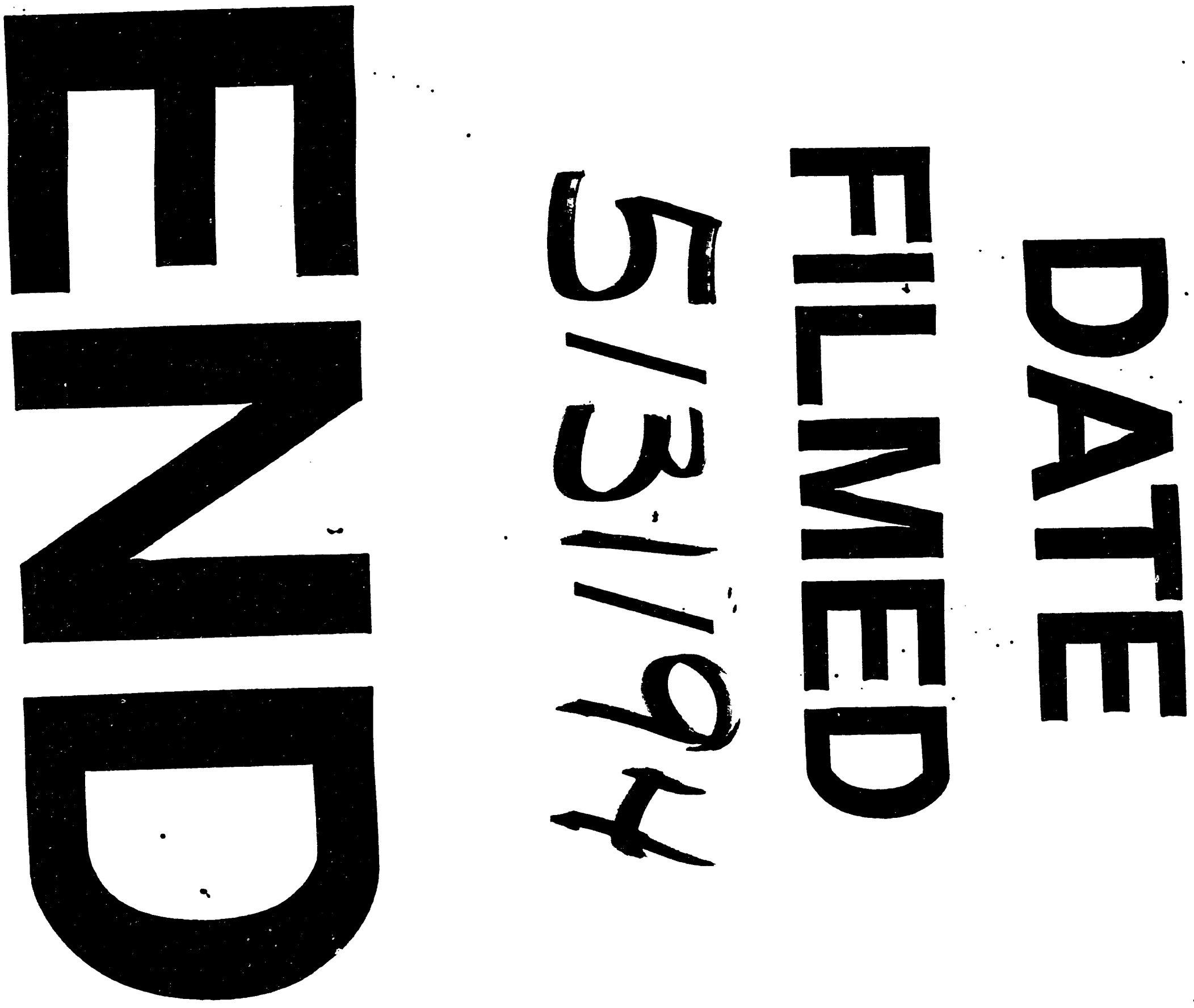
